\title{
Study of high-energy neutrinos in the FASER experiment at the LHC
}

\author{
Tomoko Ariga* for the FASER Collaboration \\ Kyushu University \\ E-mail: tomoko.ariga@cern.ch
}

FASER is a new experiment at the Large Hadron Collider (LHC) aiming to search for light, weakly interacting new particles, complementing other experiments. The particle detector will be located $480 \mathrm{~m}$ downstream of the ATLAS interaction point. In addition to searches for new particles, we also aim to study high-energy neutrinos of all flavors, as there is a huge flux of neutrinos at this location. To date, muon-neutrino cross-section data, obtained with accelerator-based neutrino beams, exist up to $350 \mathrm{GeV}$, but we are still missing data at the TeV energy scale. At LHC-FASER, the neutrino cross sections will be measured in the currently unexplored energy range between $350 \mathrm{GeV}$ and $6 \mathrm{TeV}$. In particular, tau neutrinos will be measured at the highest energy ever. Furthermore, the channels associated with heavy quark (charm and beauty) production can be studied. As a feasibility study, we performed a test run in 2018 at the proposed detector location, using a $30 \mathrm{~kg}$ lead/tungsten emulsion-based neutrino detector. We collected $12.5 \mathrm{fb}^{-1}$ of data, and expect a few tens of neutrino interactions to have been recorded in the detector. From 2021 to 2023 during Run 3 of the 14 TeV LHC, we are planning to deploy an emulsion detector with a target mass of 1.2 tons, possibly coupled with the FASER magnetic spectrometer, which would yield roughly $1,300 v_{e}, 20,000 v_{\mu}$, and $20 v_{\tau}$ interacting in the detector. Here, we present an overview of the FASER neutrino program, as well as a preliminary analysis of the 2018 test run.

European Physical Society Conference on High Energy Physics - EPS-HEP2019 -

10-17 July, 2019

Ghent, Belgium

${ }^{*}$ Speaker. 


\section{Physics motivation}

FASER $v$ [1] is a proposed detector designed to detect collider neutrinos at the highest manmade energies and to study their properties. Figure 1 shows existing constraints on neutrinonucleon charged-current (CC) scattering cross sections, together with the expected energy spectra of neutrinos interacting in the FASER $v$ detector. For all three neutrino flavors, the expected energy spectra peak at energies that are currently unconstrained. FASER $v$ will significantly extends the neutrino cross section measurements to higher energies for both electron and tau neutrinos. For muon neutrinos, FASER $v$ will fill the gap between existing measurements using accelerator experiments and IceCube [5, 6].
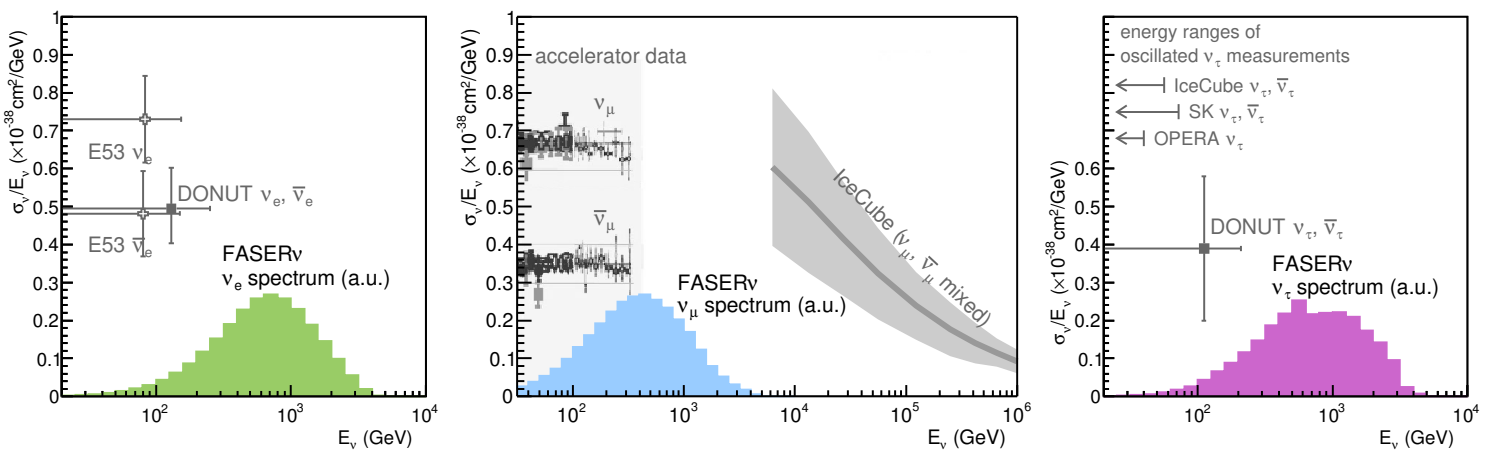

Figure 1: Existing constraints on neutrino-nucleon CC scattering cross sections ([2, 3] for $v_{e},[4,5,6]$ for $v_{\mu}$, and [3] for $v_{\tau}$ ), together with the expected energy spectra of neutrinos that interact in the FASER $v$ detector. For all three neutrino flavors, the expected energy spectra peak at energies that are currently unconstrained. For OPERA [7], SuperKamiokande [8], and IceCube [9], we indicate only the energy ranges of their respective $v_{\tau}$ cross section measurements.

\section{The FASER experiment}

FASER, the Forward Search Experiment at the Large Hadron Collider (LHC) [10], is a new experiment at the LHC with the goal of searching for light, weakly interacting particles such as dark photons and axion-like particles. Other searches for new physics at the LHC focus on high $\mathrm{p}_{T}$ (appropriate for heavy, strongly interacting particles). To complement such searches, a particle detector will be located $480 \mathrm{~m}$ downstream of the ATLAS interaction point along the beam collision axis in the unused service tunnel TI12 (Figure 2 (left)). The letter of intent [11] and technical proposal [12] for this experiment were reviewed by LHCC, and approved by CERN in March 2019.

In addition to searches for new particles, we also aim to study high-energy neutrinos of all flavors since the FASER location is also ideal for providing the first detection and studies of highenergy neutrinos produced at the LHC. Figure 2 (right) shows the expected energy spectrum of neutrinos with CC interactions at the FASER location. 

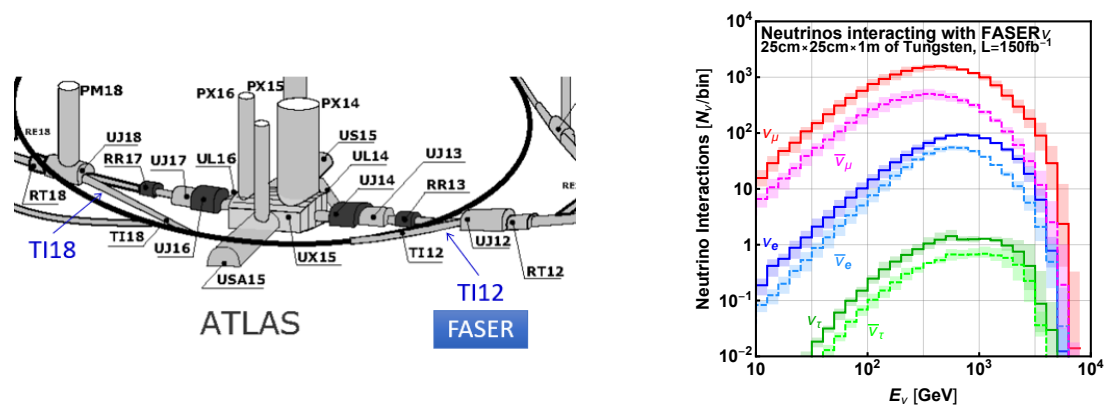

Figure 2: Left: Location of tunnels TI12 and TI18. The latter is a symmetric location on the other side of ATLAS that was also considered for FASER. Right: The energy spectrum of neutrinos with CC interactions in a 1-ton tungsten detector centered on the collision axis of the beam at the FASER location.

\section{Detector design for LHC-Run3 (2021-2023)}

The FASER $v$ neutrino detector will be located on the collision axis directly in front of the FASER main detector to maximize the expected number of neutrino interactions. Figure 3 shows a view of the neutrino-detector module. The detector is made of a repeated structure of emulsion films interleaved with 1-mm-thick tungsten plates. The emulsion film is composed of two emulsion layers, each $70 \mu \mathrm{m}$ thick, that are poured onto both sides of a $200-\mu \mathrm{m}$-thick plastic base. The whole detector contains a total of 1,000 emulsion films, with the total volume of $25 \mathrm{~cm} \times 25$ $\mathrm{cm} \times 1.35 \mathrm{~m}$, and a total tungsten mass of 1.2 tons. The total length of the detector is $1.35 \mathrm{~m}$, corresponding to 285 radiation lengths $X_{0}$ and 10.1 hadronic interaction lengths $\lambda_{\text {int }}$. For LHCRun3 (2021-2023), seven sets of emulsion detectors will be installed, with replacements installed during planned technical stops roughly every $20-50 \mathrm{fb}^{-1}$. Through LHC-Run3, about 20,000 muon neutrinos, 1,300 electron neutrinos, and 20 tau neutrinos will interact in the detector, as shown in Table 1.

\begin{tabular}{|c|c|c|}
\hline & Number of CC Interactions & Mean Energy \\
\hline$v_{e}+\bar{v}_{e}$ & $1296_{-58}^{+77}$ & $827 \mathrm{GeV}$ \\
\hline$v_{\mu}+\bar{v}_{\mu}$ & $20439_{-2314}^{+1555}$ & $631 \mathrm{GeV}$ \\
\hline$v_{\tau}+\bar{v}_{\tau}$ & $21_{-2.9}^{+3.3}$ & $965 \mathrm{GeV}$ \\
\hline
\end{tabular}

Table 1: The expected number of neutrinos with $E_{v}>100 \mathrm{GeV}$ interacting in FASER $v$ and the mean energies of the neutrinos. Here we assume a benchmark detector made of tungsten with dimensions 25 $\mathrm{cm} \times 25 \mathrm{~cm} \times 1 \mathrm{~m}$ at the 14-TeV LHC with an integrated luminosity of $\mathrm{L}=150 \mathrm{fb}^{-1}$. The uncertainties correspond to the range of predictions obtained from different Monte Carlo generators.

The neutrino event analysis will be based on readout of the full emulsion detectors by the Hyper Track Selector system [13], which is the fastest readout system at present. After processing to reconstruct the tracks in the full area of the emulsion films, we will perform a systematic analysis to locate neutrino interactions. We will classify the $v_{e}, v_{\mu}$, and $v_{\tau}$ CC interactions by identifying the $e, \mu$, and $\tau$ leptons produced in the interactions, as shown in Figure 3. We will reconstruct the neutrino energies by combining topological and kinematical variables. The resolution for reconstructing the neutrino energy is approximately $30 \%$ [1]. 


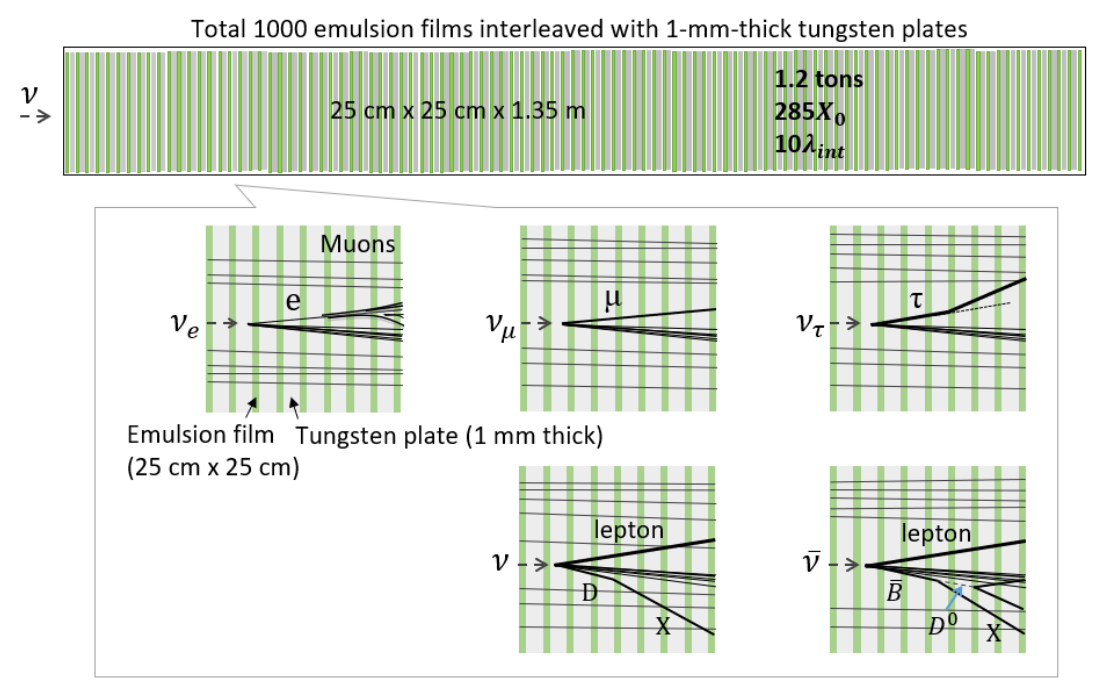

Figure 3: Conceptual design of the detector structure and the topology of various neutrino events that can be observed in the detector.

In addition, we are considering coupling FASER $v$ to the FASER spectrometer, which is located immediately downstream of FASER $v$. This can be done by means of an interface detector, an additional silicon tracker layer that interfaces FASER $v$ with the main detector, as shown in Figure 4. If events in the emulsion detector can be matched to those in the interface detector, a combined analysis will be possible, providing charge identification to enable measurements of neutrino and anti-neutrino cross sections separately, improvement of the energy resolution, and rejection of the muon-initiated neutral-hadron-interaction background.

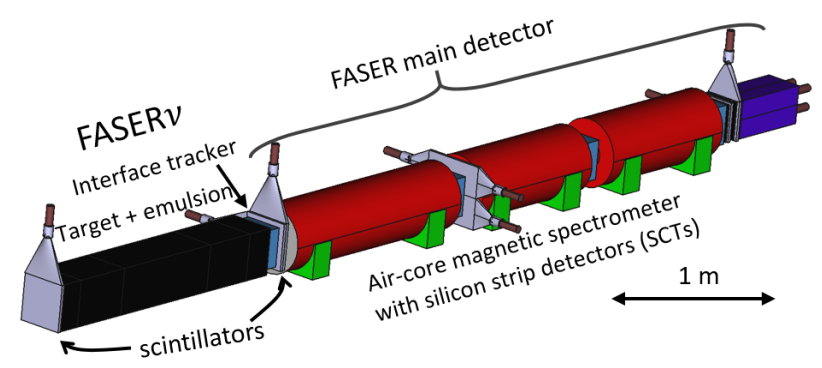

Figure 4: Plan of the detector upgrade to couple FASER $v$ to the FASER main detector.

\section{Prospect for LHC-Run3 (2021-2023)}

Figure 5 shows prospects for cross-section measurements at the 14-TeV LHC in Run3 (150 $\mathrm{fb}^{-1}$ ) using a 1.2-ton tungsten/emulsion detector. FASER $v$ will detect collider neutrinos of all three flavors and provide new measurements of their neutrino cross sections at higher energies than observed from any previous man-made source, paving the way to search for new physics effects. In addition, we will be able to study specific neutrino-interaction processes, such as charm-associated 
neutrino interactions. The FASER $v$ detector will be able to identify both charm and beauty hadrons on the basis of their decay topology, although a larger-scale experiment will be needed to observe a sizable number of beauty events. The study of neutrinos at the LHC will be a milestone, paving the way for experiments at the energy frontier to make complementary measurements in neutrino physics.
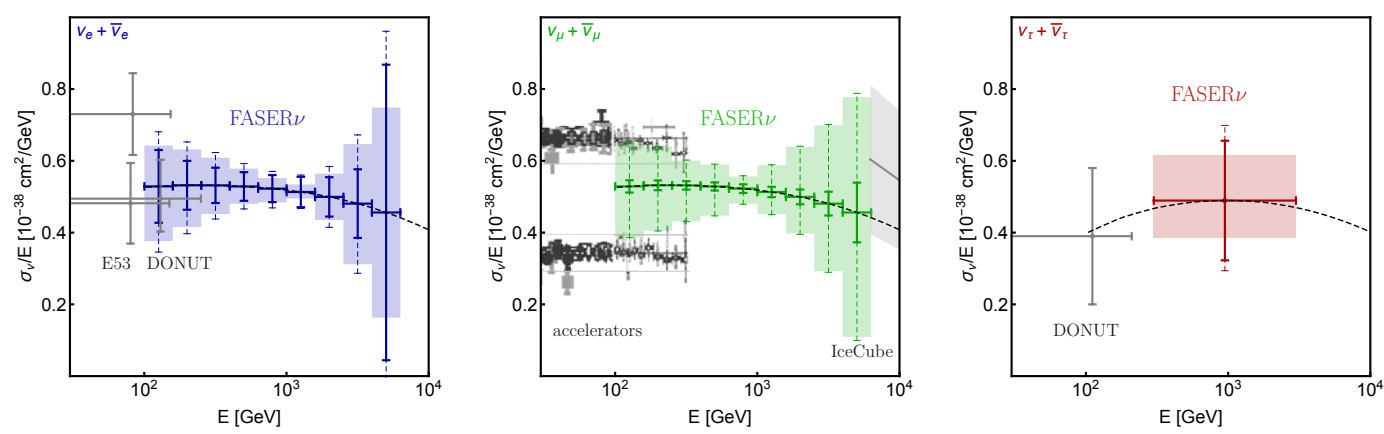

Figure 5: FASER $v$ 's estimated $v$-nucleon CC cross-section sensitivity for $v_{e}$ (left), $v_{\mu}$ (center), and $v_{\tau}$ (right) in Run 3 of the 14-TeV LHC, with an integrated luminosity of $150 \mathrm{fb}^{-1}$ in $2021-23$. Existing constraints are shown in gray. The black dashed curve is the theoretical prediction for the average DIS cross section. The solid error bars correspond to statistical uncertainties, the shaded regions show uncertainties in the neutrino-production rates corresponding to the range of predictions obtained from different MC generators, and the dashed error bars show their combination.

\section{Results from the 2018 test run}

In 2018 we performed measurements to check the detector environment. We installed emulsion detectors in the TI12 and TI18 tunnels along the collision axis of the beam or the line of sight. The flux of charged particles measured within $10 \mathrm{mrad}$ around the collision axis is $(1.9 \pm 0.2) \times$ $10^{4} \mathrm{fb} / \mathrm{cm}^{2}$, which agrees with the prediction from FLUKA simulations. The results show that the flux of high-energy particles passing through FASER $v$ is dominated by particles coming from the ATLAS interaction point.

In addition, with the aims of possible neutrino detection and gamma-ray-background measurements, we installed a 30-kg lead/tungsten emulsion-based neutrino detector in TI18. The modules were placed on the collision axis of the beam and collected $12.5 \mathrm{fb}^{-1}$ of data. During this time, we expected a few tens of neutrino interactions to have occurred in the detector. By analyzing the emulsion detector, we selected several vertex candidates from a sub-sample of the analyzed data that had no charged incoming particle (see Figure 6). These results demonstrate that emulsion detectors can work in the actual environment and that FASER $v$ has the capability to observe neutrinos from the LHC. Further detailed analysis is in progress.

\section{Summary}

At LHC-FASER, we will measure neutrino cross sections in the currently unconstrained energy range between $350 \mathrm{GeV}$ and $6 \mathrm{TeV}$. In particular, we will measure tau-neutrino cross section 

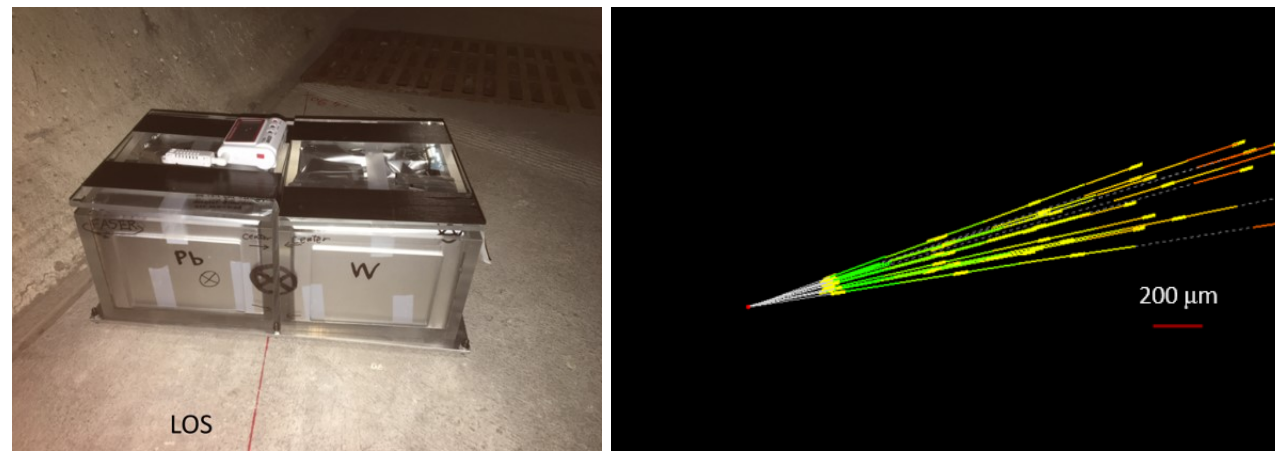

Figure 6: Left: The 30-kg pilot neutrino detector that was installed in the TI18 tunnel in 2018. It collected $12.5 \mathrm{fb}^{-1}$ of data. Right: One of vertices found in the detector with no incoming charged track. The vertex axis is compatible with the beam direction.

at the highest energy ever. For LHC-Run3 (2021-2023), we are planning to deploy a 1.2-ton tungsten/emulsion detector, possibly coupled with the FASER magnetic spectrometer, which would yield about 20,000 muon neutrinos, 1,300 electron neutrinos, and 20 tau neutrinos interacting in the detector.

As a feasibility study, we performed a test run in 2018 at the proposed detector location, using a 30-kg lead/tungsten emulsion-based neutrino detector (Figure 6). We collected $12.5 \mathrm{fb}^{-1}$ of data, for which the event analysis is currently in progress, aiming to report the first detection of neutrinos from the LHC.

\section{Acknowledgments}

The collaboration acknowledges assistance from many people, including the CERN Physics Beyond Colliders study group, the LHC Tunnel Region Experiment (TREX) working group, the CERN survey team, the CERN STI group, and the CERN Civil Engineering group. This work was supported in part by grants from the Heising-Simons Foundation (Grant Nos. 2018-1135 and 20191179 ) and the Simons Foundation (Grant No. 623683). This work is supported by JSPS KAKENHI Grant No. JP 19H01909 and a research grant from the Mitsubishi Foundation.

\section{References}

[1] FASER Collaboration, Detecting and Studying High-Energy Collider Neutrinos with FASER at the LHC, arXiv:1908.02310 [hep-ex].

[2] C. Baltayet al., $v_{\mu}-v_{e}$ Universality in Charged Current Neutrino Interactions, Phys. Rev. D 41 (1990) 2653.

[3] DONuT Collaboration, Final tau-neutrino results from the DONuT experiment, Phys. Rev. D 78 (2008) 052002.

[4] Particle Data Group Collaboration, Review of Particle Physics, Phys. Rev. D 98 no. 3, (2018) 030001.

[5] IceCube Collaboration, Measurement of the multi-TeV neutrinocross section with IceCube using Earth absorption, Nature 551 (2017) 596. 
[6] M. Bustamante and A. Connolly, Extracting the Energy-Dependent Neutrino-Nucleon Cross Section above 10 TeV Using IceCube Showers, Phys. Rev. Lett. 122 no. 4, (2019) 041101.

[7] OPERA Collaboration, Final Results of the OPERA Experiment on $v_{\tau}$ Appearance in the CNGS Neutrino Beam, Phys. Rev. Lett. 120 no. 21, (2018) 211801 [Erratum: Phys. Rev. Lett. 121, no.13, (2018) 139901].

[8] Super-Kamiokande Collaboration, Measurement of the tau neutrino cross section in atmospheric neutrino oscillations with Super-Kamiokande, Phys. Rev. D 98 no. 5, (2018) 052006.

[9] IceCube Collaboration, Measurement of Atmospheric Tau Neutrino Appearance with IceCube DeepCore, Phys. Rev. D 99 no. 3, (2019) 032007.

[10] J. L. Feng, I. Galon, F. Kling, and S. Trojanowski, ForwArd Search ExpeRiment at the LHC, Phys. Rev. D97 no. 3, (2018) 035001.

[11] FASER Collaboration, Letter of Intent for FASER: ForwArd SearchExpeRiment at the LHC, arXiv:1811.10243 [physics.ins-det]. https://cds.cern.ch/record/2642351. Submitted to the CERN LHCC on 18 July 2018.

[12] FASER Collaboration, Technical Proposal for FASER: ForwArd SearchExpeRiment at the LHC, arXiv:1812.09139 [physics.ins-det]. http://cds.cern.ch/record/2651328. Submitted to the CERN LHCC on 7 November2018.

[13] M. Yoshimoto, T. Nakano, R. Komatani, and H. Kawahara, Hyper-track selector nuclear emulsion readout system aimed at scanning an area of one thousand square meters, PTEP 2017 no. 10 (2017) 103 H01. 